\title{
Technology update: intracardiac echocardiography a review of the literature
}

This article was published in the following Dove Press journal:

Medical Devices: Evidence and Research

27 May 2015

Number of times this article has been viewed

\author{
Nicola Vitulano \\ Vincenzo Pazzano \\ Gemma Pelargonio \\ Maria Lucia Narducci \\ Institute of Cardiology, \\ Department of Cardiovascular \\ Sciences, Catholic University of \\ the Sacred Heart, Rome, Italy
}

Correspondence: Maria Lucia Narducci Institute of Cardiology, Department of Cardiovascular Sciences, Catholic University of the Sacred Heart, Largo Agostino Gemelli, 8, 00168 Rome, Italy Tel +3906 30I5 4I87

Fax +3906305 5535

Email lianarducci@yahoo.it

\begin{abstract}
The development of new imaging tools helps in better investigation of cardiac structures and function by showing detailed images during interventional procedures. Intracardiac echocardiography plays a pivotal role as an intraoperative real-time imaging tool during invasive cardiac procedures. Initially, this echocardiographic technique was particularly useful when transthoracic image quality was insufficient and to avoid general anesthesia for transesophageal imaging. Nowadays, intracardiac echocardiography is routinely used in several cardiac invasive laboratories to support several types of procedures, such as extraction and implantation of cardiac devices, electrophysiological mapping, ablation, and endomyocardial biopsies. This review gives an overview of the basic principles of intracardiac echocardiography and examines its applications in the different settings of invasive cardiology.
\end{abstract}

Keywords: ICE, cardiovascular imaging, electrophysiology, invasive cardiology

\section{Introduction}

Ultrasound devices have had a great technological development in the past decades, with growing diagnostic accuracy and a broadening range of clinical applications, being now an essential tool for evaluation and treatment of various diseases. Particularly, in the field of interventional cardiology, the introduction of intracardiac echocardiography (ICE), with its power to visualize cardiac structures and blood flow from the inside, has brought a very useful support in several procedures. First described in $1981,{ }^{1}$ ICE is now widely used in cardiac catheterization laboratories and in electrophysiology as an advanced imaging approach, allowing for an accurate percutaneous placement of catheters, closure devices, and prosthetic heart valves. This echocardiographic technique has contributed to increased safety and reduced radiation exposure time in complex procedures. Compared with transesophageal echocardiography (TEE), ICE has the same image quality but a broader view due to the major maneuverability of the catheter and important advantages both for the operator and for the patient. Unlike TEE, which requires the presence of a dedicated sonographer, the ICE catheter could be controlled by the cardiologist performing the procedure. It is also better tolerated by the patient and does not require general anesthesia. ${ }^{2}$ On the other hand, specific training is essential for the cardiologist to become confident with this technique. The principal disadvantage is represented by its additional cost. This article provides a comprehensive review of the current technology for ICE and its growing applications in the setting of interventional cardiology. 


\section{Technical features}

ICE uses a catheter-based steerable ultrasound probe that is introduced into the right heart chambers displaying cardiac structures from the inside (Figure 1A). The phased-array catheter system (Acuson AcuNav ultrasound catheter, Siemens, Mountain View, CA, USA) presents a handle component and a catheter provided with a 64-element transducer, scanning longitudinally and providing a $90^{\circ}$ sector image, with a frequency ranging 5-10 MHz and a tissue penetration that can reach approximately $15 \mathrm{~mm}$. This system has replaced the older rotational catheter, which provided images in a $360^{\circ}$ radial plane with poor spatial resolution $(5 \mathrm{~cm})$. The phased-array catheter is characterized by a higher tissue penetration, giving the possibility to visualize left cardiac structures with the probe located in the right chambers. Other features are represented by optimal maneuverability, with the possibility of anterior/posterior and left/right deflection of the catheter and a blockage system holding the probe in the desired position during the procedure (Figure 1B). The system includes the presence of full-color Doppler capabilities. The probe used for ICE has a diameter that varies from $8 \mathrm{Fr}$ to $10 \mathrm{Fr}$, with a length of $90 \mathrm{~cm}$, and is introduced with a vascular approach (femoral or jugular) requiring only local anesthesia and is thus well tolerated.

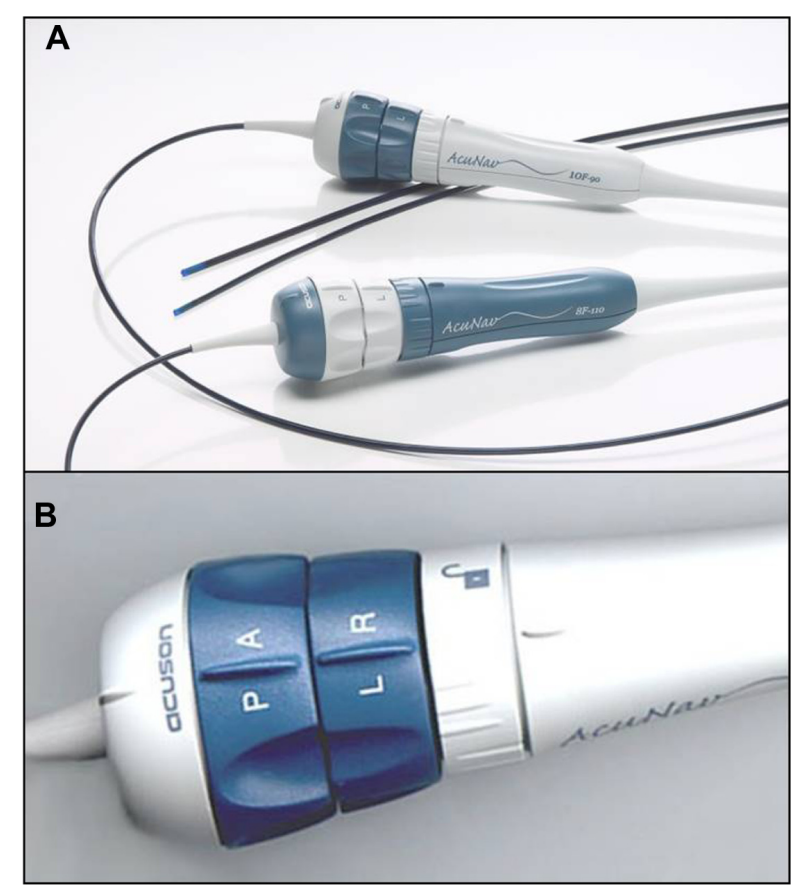

Figure I Ultrasound probes.

Notes: The catheter-based steerable ultrasound probe that is introduced into the right heart chambers displaying cardiac structures from the inside (A) with optimal manoeuvrability with the possibility of catheter anterior/posterior and left/right deflection and a blockage system holding the probe in the desired position during the procedure (B).
The transducer can be variably positioned to obtain optimal imaging during cardiac procedures. In the so-called "home view", the probe is located in the middle of the right atrium, is slightly deflected anteriorly, and shows the right heart from the atrium toward the right ventricle (Figures $2 \mathrm{~A}$ and $3 \mathrm{~A}$ ). With a $30^{\circ}$ clockwise rotation, it is possible to visualize aortic and tricuspid valves, right ventricle outflow tract, and pulmonary artery (Figures $2 \mathrm{~B}$ and $3 \mathrm{~B}$ ); while turning $60^{\circ}-70^{\circ}$ clockwise from the home view, without deflection, it is possible to view the interatrial septum, left atrium (LA) and left atrial appendage (LAA), mitral valve (MV), and left ventricle (Figures 2C and 3C). However, for a better visualization of the fossa ovalis (FO), it is necessary to turn beyond MV, with the catheter deflected posteriorly, exercising a right/left deflection (Figures 2D and 3D). By a further clockwise rotation of $90^{\circ}-100^{\circ}$, without deflection, the left pulmonary veins (PVs) are visualized (Figures 4A and 5A). Color-Doppler imaging could be used for a noninvasive imaging of blood flow through veins and valves. The view is completed with the display of the right PVs obtained by shifting and rotating the catheter $150^{\circ}-180^{\circ}$ clockwise (Figures $4 \mathrm{~B}$ and $5 \mathrm{~B}$ ): a longitudinal view of the PVs is obtained by deflecting the probe on a left/ right plane. From the home view in the middle right atrium, it is possible to deflect anteriorly and move the catheter forward in the right ventricle, then turn clockwise to show the interventricular septum (Figures $4 \mathrm{C}$ and 5C), and further deflect the probe to change from a long-axis to a short-axis view (Figures 4D and 5D).

\section{Clinical applications}

Transesophageal echocardiography (two-dimensional and RT three-dimensional [3D] TEE) is widely accepted for intraprocedural imaging modalities for many cardiac interventions. In the recent years, ICE has been increasingly used in a number of interventional procedures such as device closure of interatrial communications and ablation of cardiac arrhythmias. ICE may provide potential advantages, such as avoidance of general anesthesia and reduction of radiation exposure. However, the additional cost and the need for specific operator skills remain its limitations. Currently, ICE can be considered as an alternative or supplementary guiding tool in cardiac interventional procedures, such as in percutaneous valvular implantation and valvuloplasty, lead extraction, and endomyocardial biopsy. In order to improve safety and outcome of these procedures, randomized multicenter trials regarding ICE guidance are needed.

The main interventional procedures performed with ICE systems are the following: 


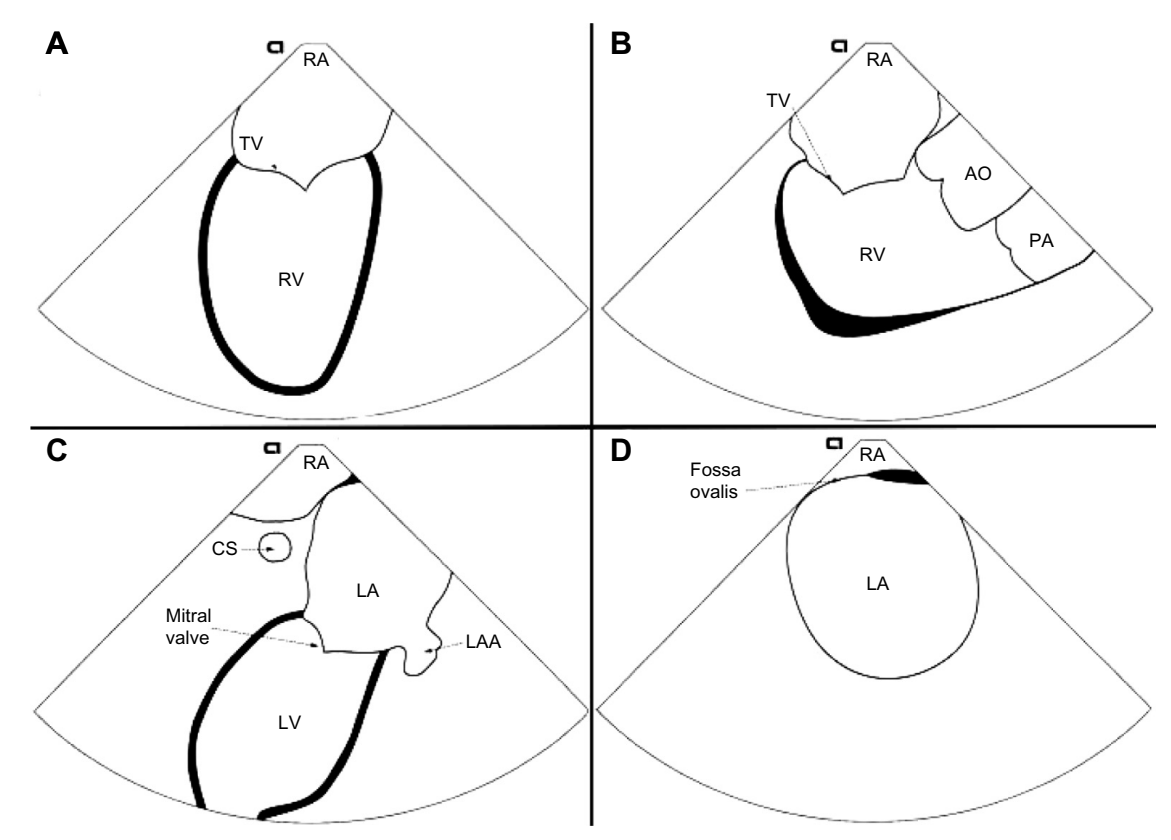

Figure 2 Cardiac chambers by intracardiac echocardiography.

Notes: (A-D) a schematic view of cardiac chambers from the so called home-view in the middle of the right atrium, through slightly deflected anteriorly and subsequent clockwise rotation in order to display the other cardiac structures.

Abbreviations: AO, aorta; CS, coronary sinus; LA, left atrium; LAA, left atrial appendage; LV, left ventricle; MV, mitral valve; PA, pulmonary artery; RA, right atrium; RV, right ventricle; TV, tricuspid valve.

1. transseptal puncture and interatrial defect closure

2. percutaneous valvular implantation and valvuloplasty

3. ablation of complex arrhythmias

4. lead extraction and device-related endocarditis

5. endomyocardial biopsy.

\section{Transseptal puncture and interatrial communication closure}

Transseptal access to the LA is a prerequisite for several procedures: atrial fibrillation (AF) ablation, MV valvuloplasty, and LAA closure. ICE has been demonstrated to be particularly
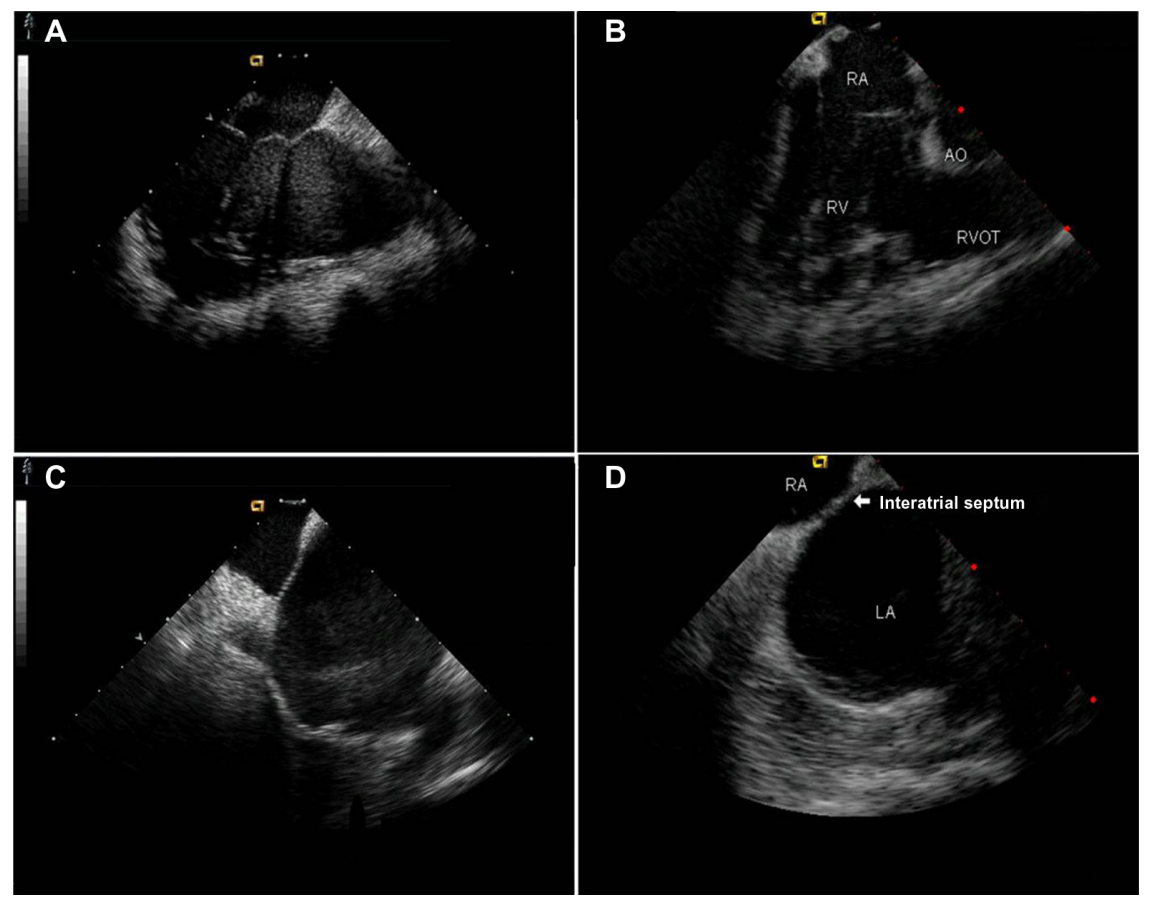

Figure 3 Real-time acquisition by intracardiac echocardiography.

Notes: (A-D) comparison of the imaging from the schematic view to real time acquisition.

Abbreviations: AO, aorta; LA, left atrium; RA, right atrium; RV, right ventricle; RVOT, right ventricle outflow tract. 


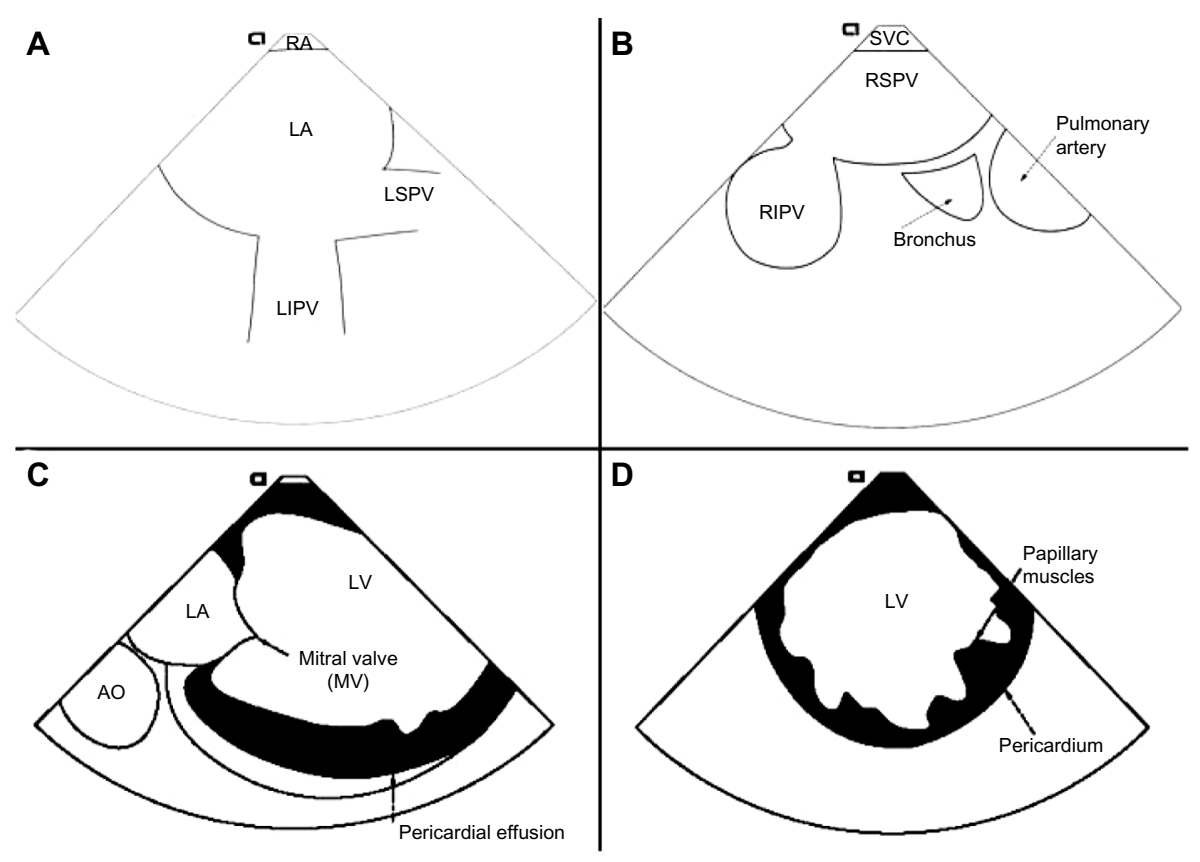

Figure 4 Cardiac chambers and vessels by intracardiac echocardiography.

Notes: (A-D) from the view for the visualization of fossa ovalis, we can visualize the left PVs by a further clockwise rotation of $90^{\circ}-100^{\circ}$ degrees. The view is completed with the display of the right PVs obtained shifting and rotating the catheter $150^{\circ}-180^{\circ}$ degrees clockwise: a longitudinal view of the veins is warranted deflecting the probe on a left/right plane. From the home view in middle RA it is possible to deflect anteriorly and move the catheteter forward in the right ventricle, then turning clockwise to show the interventricular septum, and further deflect the probe to change from a long-axis to a short-axis view.

Abbreviations: RA, right atrium; RV, right ventricle; LA, left atrium; LV, left ventricle; LIPV, left inferior pulmonary vein; LSPV, left superior pulmonary vein; RIPV, right inferior pulmonary vein; RSPV, right superior pulmonary vein; SVC, superior vena cava.

useful to achieve a safe transseptal puncture (especially in the presence of anatomical anomalies of the interatrial septum). The localization of the needle tip position on the atrial septum and the clear visualization of the "tenting effect" on the FO are
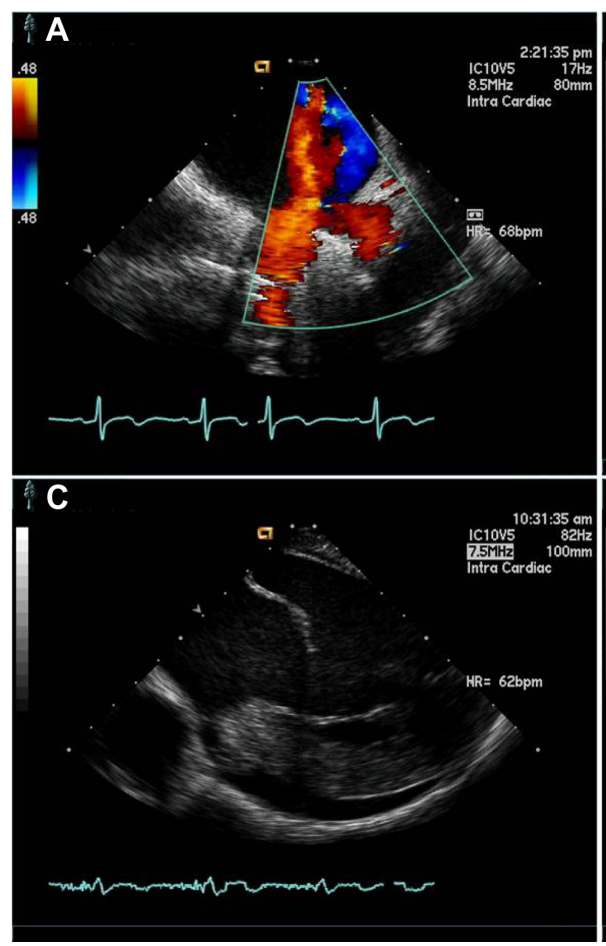

important advantages of this technique. ${ }^{3}$ The usefulness of ICE is evident above all in patients with implanted devices for patent foramen ovale or atrial septal defect closure, in which transseptal puncture may prove to be particularly challenging. ${ }^{4,5}$ Venous

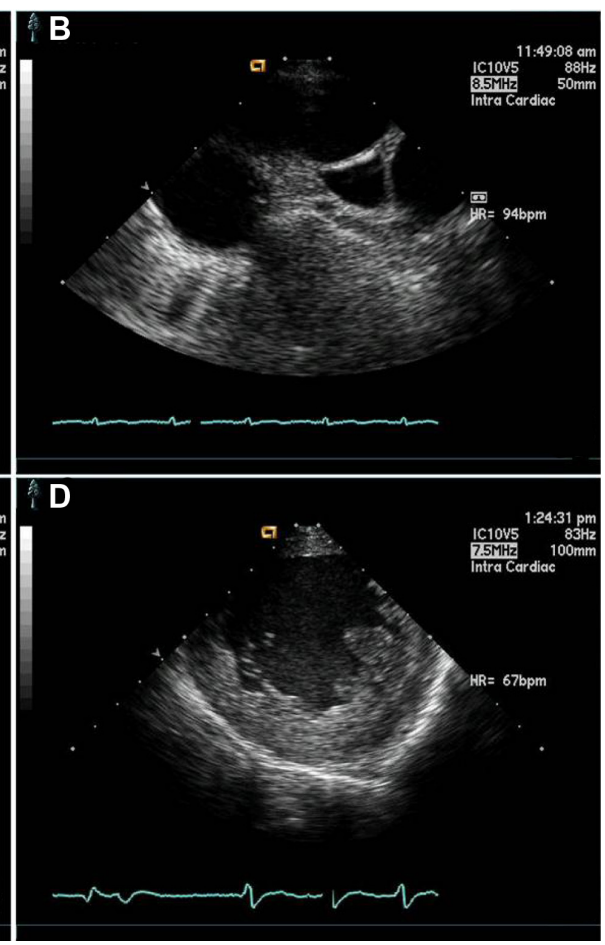

Figure 5 Real-time acquisition by intracardiac echocardiography.

Notes: (A-D) comparison of the imaging from the schematic view to real time acquisition. 
ICE was compared in terms of efficacy and safety with arterial (intra-aortic) ICE in a recent study, with no major complications with the use of aortic ICE. This represents a valid alternative to venous ICE and facilitates transseptal puncture in patients with mitral stenosis undergoing valvuloplasty. ${ }^{6}$

The detailed visualization of the interatrial septum provided by ICE is also used to guide device closure of interatrial communications. ${ }^{7}$ ICE was shown to be superior to TEE in terms of fluoroscopy time and reduction in total procedure time, as well as procedure tolerability. ${ }^{8}$ Data regarding its use in children are limited. Recent findings of a retrospective study, analyzing all cases in which ICE was used during atrial septal defect or patent foramen ovale closure in 115 patients $\leq 21$ years of age, show that ICE can be performed safely and efficaciously in a large cohort of children and adolescents undergoing percutaneous device closure of interatrial defects. ${ }^{9}$ In this study, atrial septal defect measurements obtained with ICE correlate well with those obtained preprocedurally with transthoracic echocardiography. Moreover, ICE was proved to identify with major accuracy the absence or deficiency of critical septal rims.

Some authors have also proposed the use of ICE to guide LAA closure. However, there are still few data available and TEE continues to be the first choice imaging approach used for this procedure. ${ }^{10,11}$

\section{Percutaneous valvular implantation and valvuloplasty}

Echocardiographic imaging is an essential component of successful transcatheter aortic valve replacement. Although TEE is actually the imaging modality of first choice as a guide to such procedure, its limitations are considerable. The most important are represented by the need for general anesthesia with endotracheal intubation in most cases and the interference generated by the probe to fluoroscopic views. Thus, ICE represents a useful alternative, especially considering the recent availability of a new volumetric 3D intracardiac ultrasound system (Siemens Acuson AcuNav V ultrasound catheter, Mountain View, CA, USA), improving the visualization of valvular, perivalvular, and aortic structures. ${ }^{12}$ Moreover, ICE may be useful in a new percutaneous approach to aortic valve disease such as retrograde double-balloon aortic valvuloplasty, a recently developed method for decreasing the transvalvular gradient alternative to the conventional single-balloon technique. ${ }^{13}$

\section{Ablation of complex arrhythmias}

ICE has had a major impact on electrophysiology procedures. Its contribution to the identification and targeting of arrhythmic substrates, especially when integrated to other
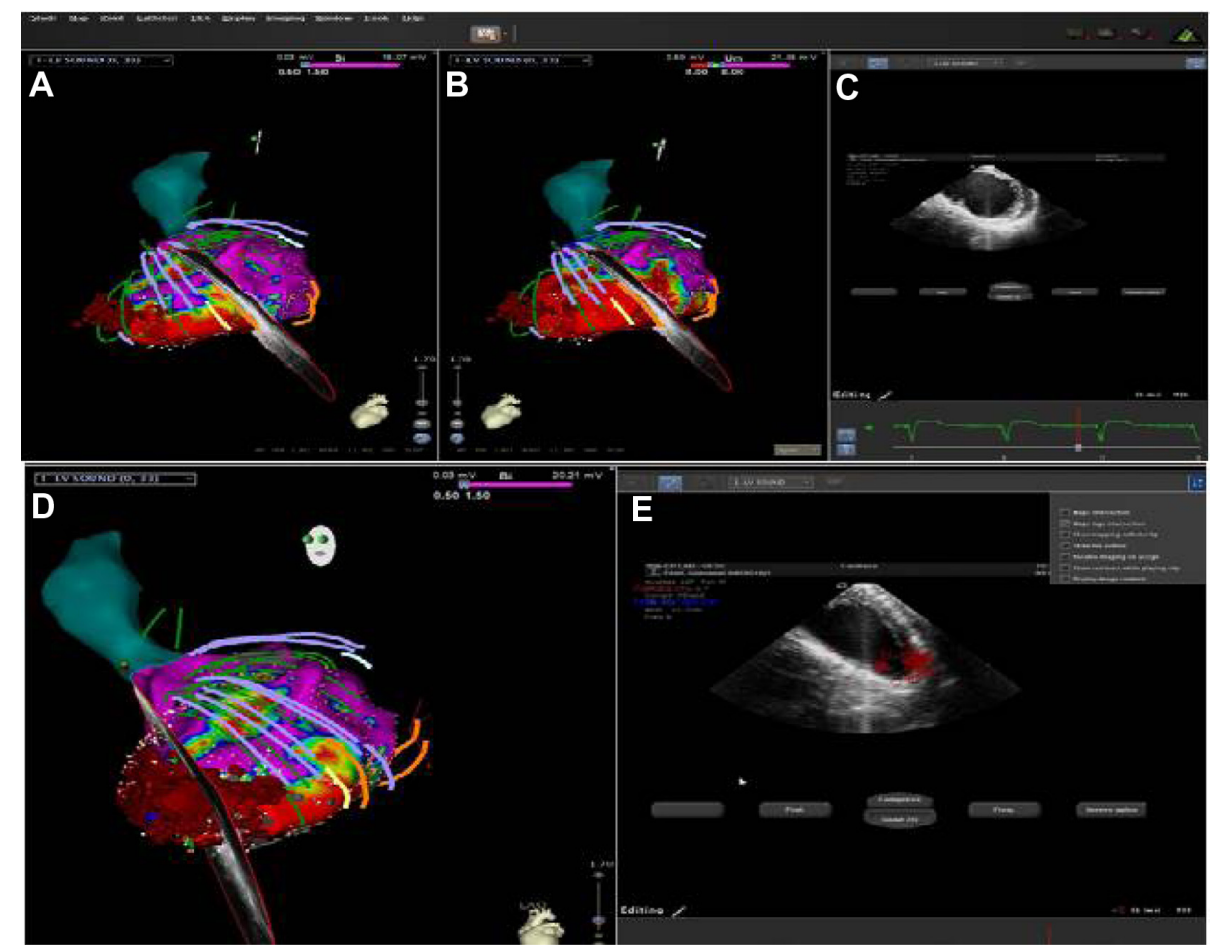

Figure 6 Integration of intracardiac echocardiography with ventricular electronanatomic mapping.

Notes: (A, B, D) Integration of intracardiac echocardiography with bipolar and unipolar left ventricular electroanatomic mapping. (C, E) Intracardiac echocardiographic views of all left ventricular layers. ICE gives an important support during ablation procedures with information about the contact between the mapping/ablating catheter and the myocardial tissue, indicating the sites where RF energy has been delivered (red tags). Comparison with electroanatomic maps may show the anatomical features of areas of potential interest and provide a real time continuous monitoring and surveillance of potential procedural complications.

Abbreviations: ICE, intracardiac echocardiography; RF, radiofrequency. 
imaging techniques (especially electroanatomic mapping), has progressively led to a wide use of this tool in the electrophysiology laboratory. It provides information about the contact between the mapping/ablating catheter and the myocardial tissue, showing the anatomical features of areas of electrical interest. It can also guide the catheters in complex anatomic settings such as in patients with congenital heart disease and warrants a real-time continuous monitoring and surveillance of potential procedural complications (Figure 6). ${ }^{14-17}$ All these features represent an important support during ablation procedures.

A transseptal access to the LA is required to perform AF ablation. The role of ICE in guiding transseptal puncture has already been discussed. In AF ablation in particular, ICE allows real-time direct imaging of the FO, posterior atrial wall, and aorta, helping the operator to choose with more precision the puncture site (eg, posterior region of the FO) in order to achieve maximum catheter maneuverability during mapping and ablation. Visualization of the tenting effect, position of sheath and needle, and detection of microbubbles in LA after injection of contrast dye indicating successful puncture are other useful indicators. For these reasons and for its role in guiding catheter placement during mapping and ablation, ICE gives the possibility to perform an AF ablation procedure abolishing the use of fluoroscopy. ${ }^{16}$ Usually integrated to other imaging techniques, ICE can give further information about the individually variable anatomy of PVs, permitting identification of their number, position, and size, as well as the presence of a common ostium and evaluation of the position, dimensions, and shape of the LAA, as well as its spatial relationship with the left PVs. ${ }^{17}$ It also allows precise real-time visualization of the mapping/ablating catheter position in relation to these structures and can be used in addition to electrophysiological indices such as impedance monitoring to identify sites that are safe for energy delivery to obtain PV isolation. ${ }^{18,19}$ Furthermore, titration of radiofrequency energy based on visualization of microbubbles by ICE has been demonstrated to improve long-term outcome and reduce $\mathrm{PV}$ stenosis after AF ablation. ${ }^{20} \mathrm{ICE}$ has also been proposed as a means to investigate the presence of a thrombus in the LAA, but evidence still shows the superiority of TEE for this purpose. ${ }^{21}$ Finally, ICE plays an important role in monitoring for possible intraprocedural complications, the most threatening being cardiac perforation with consequent pericardial effusion and tamponade, thromboembolism, and esophageal injury. In all these cases, an early detection warranted by ICE will lead to a prompt intervention resulting in a better final outcome. ${ }^{22}$ For example, the identification of an initial pericardial effusion will lead to the immediate interruption of anticoagulation and the performance of pericardiocentesis (if needed) before the occurrence of cardiac tamponade. In case of echographic evidence of intracardiac thrombus formation on catheters or endocardial lesion sites, an additional anticoagulation dose could be immediately administered preventing embolic complications. ICE is also used during other supraventricular arrhythmia ablations, especially in disorders for which the ablation approach is typically anatomically based, like atrial tachycardias originating from the crista terminalis ${ }^{23}$ or challenging atrial flutters, ${ }^{24}$ for example, in the presence of prominent pectinate muscles and subeustachian pouches or in patients with complex anatomy due to congenital heart disease. ${ }^{25}$

The contribution of ICE described in AF ablation procedures can be appreciated as well in ventricular arrhythmia ablations: the ability to visualize precise catheter position has proven to be very useful in the ablation of ventricular tachycardia (VT) originating close to delicate cardiac structures such as coronary ostia (in idiopathic VTs from Valsalva sinuses) or arteries (in epicardial ablations) or from areas in which getting a stable catheter-tissue contact can be challenging, like papillary muscles. As already discussed about AF ablation, continuous monitoring provided by ICE is very useful to prevent or rapidly treat potential complications in VT ablations too. However, the peculiar contribution of ICE in VT ablation procedures is represented by its role in the identification of the arrhythmic substrate, especially if combined with electroanatomic mapping techniques. Most VTs originate from myocardial scars secondary to ischemia and infarction or from the border zones between scars and adjacent tissue. Thus, the identification of these akinetic areas as arrhythmogenic substrate is a useful endpoint for a successful ablation. The identification of the akinetic and diskinetic areas or aneurismatic dilatation by ICE could give additional information ${ }^{26,27}$ compared to traditional electroanatomic mapping system, defining not only the relevant arrhythmogenic myocardial areas but also the wall thickness before ablation. ${ }^{27,28}$ This imaging tool could be helpful also in the context of epicardial ablation of $\mathrm{VT}^{28}$

\section{Lead extraction and device-related endocarditis}

The incidence of device-related infectious complications is gradually increasing due to the growth of the number of patients with implantable pacemakers (PMs) or implantable cardioverter defibrillators (ICDs). The infection can be limited to the device pocket causing local erythema, suppuration, or externalization 
of the generator, but it may also affect leads with consequent risk of sepsis, valvular (tricuspid) endocarditis, and pulmonary septic embolism with dissemination of the infectious disease. The diagnosis of infective endocarditis is made using the modified Duke criteria, mainly based on echocardiographic and blood culture results. In device-related endocarditis in particular, extraction of the entire system is required to eradicate the infection. Moreover, the presence of intracardiac vegetations has an important impact in terms of prognosis and treatment, requiring a prolonged antibiotic therapy compared to device infection without the involvement of intracardiac structures. Thus, ICE plays a key role in the diagnosis and treatment of device-related infections, providing an excellent imaging of right-sided PM/ICD leads, prosthetic valves, and cardiac structures, proven to be superior to TEE in detecting signs of infectious involvement, guiding the procedures of lead extraction and monitoring for complications..$^{29,30}$

\section{Endomyocardial biopsy}

In regard to endomyocardial biopsy, ICE is an optimal imaging tool to guide the procedure, with direct visualization of the biopsy zone and real-time monitoring of potential adverse effects. Although right ventricular endomyocardial biopsy is routinely performed in many cardiac catheterization laboratories, it remains a potentially harmful procedure. Thus, the support of ICE could be particularly helpful. During endomyocardial biopsy, ICE guides the choice of the optimal sampling site, making at the same time possible to monitor for complications. This is of remarkable importance if the procedure involves high-risk structures like papillary muscle and/or cardiac wall affected by pathological processes that reduce thickness and modify tissue architecture (eg, the fibrofatty replacement typical of arrhythmogenic right ventricular cardiomyopathy). Moreover, directing a bioptome to an intracardiac mass is extremely difficult with fluoroscopic or echocardiographic guidance. The use of ICE facilitates biopsies of such lesions and, also imaging them with high resolution, plays a fundamental role in the differential diagnosis of intracardiac neoplasms. ${ }^{31}$ Several case reports highlight the importance, the usefulness, and the safety of ICE during the diagnostic pathway of cardiac tumors. ${ }^{32,33}$

\section{Future perspectives}

In the next future, clinical applications of ICE are expected to expand. As already discussed, the experience of its use in LAA closure is still limited, but is probably going to grow in the next years. Similarly, there are still several procedures in which the imaging technique is alternatively chosen between ICE and TEE, according to the operator's experience and preference. "ICE-guided" interventional procedures include valvuloplasty and other more complex percutaneous valvular interventions, such as transcatheter aortic valve implantation and percutaneous MV repair. Considering its potential and its many applications, ICE will certainly play a fundamental role in the hybrid catheterization labs that are developing in the most advanced cardiology centers.

Regarding technological features, future challenges include further optimization of image resolution and ultrasound tissue penetration. Integration between ICE and electroanatomical voltage mapping has been discussed. Extension of the integration with other imaging techniques could lead to merging of echographic images with those obtained from cardiac magnetic resonance or CT. Finally, a further development of 3D ICE is expected. This could be particularly useful during several procedures including leads extraction. In this setting, a 3D real-time imaging of the intracardiac structures will allow direct visualization of the anatomical relationship between electrocatheters and tricuspid leaflets and, consequently, identification of lead and/or valvular vegetations.

\section{Conclusion}

ICE provides important information during invasive cardiac procedures. During the examination, the patient is awake, making the procedure more tolerable not requiring general anesthesia and also making the patient able to collaborate with the operator (informing about every symptoms, making Valsalva maneuver if requested, etc). ICE provides continuous and real-time monitoring of possible complications (eg, pericardial effusion or intracardiac/catheter thrombosis). Further advantages are represented by the reduction of procedural time and fluoroscopy time. Moreover, this system could be directly used by interventional operators without the presence of a dedicated sonographer. Its main disadvantage is represented by the additional cost, but a focused cost-effectiveness analysis should be performed. Regarding this issue, the reduction of procedural time, radiation exposure, and the early monitoring of complications achieved with ICE could be associated with an important long-term cost reduction. This feature, together with the continuous development of technology to further optimize the imaging of cardiac structures (eg, 3D imaging), defines ICE as a promising diagnostic tool for multiple interventional procedures.

\section{Acknowledgment}

The authors thank professor Andrea Natale for his guidance in intracardiac echocardiography during EP procedures. The 
authors also thank Federico Pintus and Lorenzo Vitulano for providing technical help for imaging. All authors declare that written informed consent was obtained from the patients for publishing the accompanying images.

\section{Disclosure}

The authors report no conflicts of interest in this work.

\section{References}

1. Glassman E, Kronzon I. Transvenous intracardiac echocardiography. Am J Cardiol. 1981;47(6):1255-1259.

2. Boccalandro F, Baptista E, Muench A, Carter C, Smalling RW. Comparison of intracardiac echocardiography versus transesophageal echocardiography guidance for percutaneous transcatheter closure of atrial septal defect. Am J Cardiol. 2004;93(4):437-440.

3. Citro R, Ducceschi V, Salustri A, Santoro M, Salierno M, Gregorio G. Intracardiac echocardiography to guide transseptal catheterization for radiofrequency catheter ablation of left-sided accessory pathways: two case reports. Cardiovasc Ultrasound. 2004;2:20.

4. Santangeli P, Di Biase L, Burkhardt JD, et al. Transseptal access and atrial fibrillation ablation guided by intracardiac echocardiography in patients with atrial septal closure devices. Heart Rhythm. 2011;8(11): 1669-1675.

5. Li X, Wissner E, Kamioka M, et al. Safety and feasibility of transseptal puncture for atrial fibrillation ablation in patients with atrial septal defect closure devices. Heart Rhythm. 2014;11(2):330-335.

6. Akkaya E, Vuruskan E, Zorlu A, et al. Aortic intracardiac echocardiography-guided septal puncture during mitral valvuloplasty. Eur Heart J Cardiovasc Imaging. 2014;15(1):70-76.

7. Mullen MJ, Dias BF, Walker F, Siu SC, Benson LN, McLaughlin PR. Intracardiac echocardiography guided device closure of atrial septal defects. J Am Coll Cardiol. 2003;41(2):285-292.

8. Bartel T, Konorza T, Arjumand J, et al. Intracardiac echocardiography is superior to conventional monitoring for guiding device closure of interatrial communications. Circulation. 2003;107(6):795-797.

9. Medford BA, Taggart NW, Cabalka AK, et al. Intracardiac echocardiography during atrial septal defect and patent foramen ovale device closure in pediatric and adolescent patients. J Am Soc Echocardiogr. 2014;27(9):984-990.

10. Fassini G, Russo AD, Conti S, Tondo C. An alternative transseptal intracardiac echocardiography strategy to guide left atrial appendage closure: the first described case. J Cardiovasc Electrophysiol. 2014;25(11):1269-1271.

11. Ternacle J, Lellouche N, Deux JF, et al. Left atrial appendage closure monitoring without sedation: a pilot study using intracardiac echocardiography through the oesophageal route. EuroIntervention. In press 2014.

12. Kadakia MB, Silvestry FE, Herrmann HC. Intracardiac echocardiographyguided transcatheter aortic valve replacement. Catheter Cardiovasc Interv. In press 2014.

13. Inohara T, Hayashida K, Fukuda K. Double balloon aortic valvuloplasty in TAVI era: insight from intracardiac echocardiography and multidetector computed tomography findings. J Invasive Cardiol. 2014;26(7): E95-E97.

14. Khaykin Y, Skanes A, Whaley B, et al. Real-time integration of 2D intracardiac echocardiography and $3 \mathrm{D}$ electroanatomical mapping to guide ventricular tachycardia ablation. Heart Rhythm. 2008;5(10): 1396-1402.

15. Rausch P, Manfai B, Varady E, Simor T. Radiofrequency catheter ablation of left ventricular outflow tract tachycardia with the assistance of the CartoSound system. Europace. 2009;11(9):1248-1249.

16. Ferguson JD, Helms A, Mangrum JM, et al. Catheter ablation of atrial fibrillation without fluoroscopy using intracardiac echocardiography and electroanatomic mapping. Circ Arrhythm Electrophysiol. 2009;2(6): 611-619.
17. Kean AC, Gelehrter SK, Shetty I, Dick M 2nd, Bradley DJ. Experience with CartoSound for arrhythmia ablation in pediatric and congenital heart disease patients. J Interv Card Electrophysiol. 2010;29(2):139-145.

18. Daccarett M, Segerson NM, Günther J, et al. Blinded correlation study of three-dimensional electro-anatomical image integration and phased array intra-cardiac echocardiography for left atrial mapping. Europace. 2007;9(10):923-926.

19. Vaseghi M, Cesario DA, Valderrabano M, et al. Impedance monitoring during catheter ablation of atrial fibrillation. Heart Rhythm. 2005;2: 914-920.

20. Marrouche NF, Martin DO, Wazni O, et al. Phased-array intracardiac echocardiography monitoring during pulmonary vein isolation in patients with atrial fibrillation: impact on outcome and complications. Circulation. 2003;107(21):2710-2716.

21. Saksena S, Sra J, Jordaens L, et al; ICE-CHIP Investigator Study Group. A prospective comparison of cardiac imaging using intracardiac echocardiography with transesophageal echocardiography in patients with atrial fibrillation: the intracardiac echocardiography guided cardioversion helps interventional procedures study. Circ Arrhythm Electrophysiol. 2010;3:571-577.

22. Aldhoon B, Wichterle D, Peichl P, Čihák R, Kautzner J. Complications of catheter ablation for atrial fibrillation in a high-volume centre with the use of intracardiac echocardiography. Europace. 2013;15: 24-32.

23. Kalman JM, Olgin JE, Karch MR, Hamdan M, Lee RJ, Lesh MD. "Cristal tachycardias": origin of right atrial tachycardias from the crista terminalis identified by intracardiac echocardiography. J Am Coll Cardiol. 1998;31:451-459.

24. Morton JB, Sanders P, Davidson NC, Sparks PB, Vohra JK, Kalman JM. Phased array intracardiac echocardiography for defining cavotricuspid isthmus anatomy during radiofrequency ablation of typical atrial flutter. J Cardiovasc Electrophysiol. 2003;146:591-597.

25. Sherwin ED, Triedman JK, Walsh EP. Update on interventional electrophysiology in congenital heart disease: evolving solutions for complex hearts. Circ Arrhythm Electrophysiol. 2013;6(5):1032-1040.

26. Jongbloed MR, Bax JJ, van der Burg AE, Van der Wall EE, Schalij MJ. Radiofrequency catheter ablation of ventricular tachycardia guided by intracardiac echocardiography. Eur J Echocardiogr. 2004;5(1): $34-40$.

27. Hussein A, Jimenez A, Ahmad G, et al. Assessment of ventricular tachycardia scar substrate by intracardiac echocardiography. Pacing Clin Electrophysiol. 2014;37(4):412-421.

28. Bala R, Ren JF, Hutchinson MD, et al. Assessing epicardial substrate using intracardiac echocardiography during VT ablation. Circ Arrhythm Electrophysiol. 2011;4(5):667-673.

29. Narducci ML, Pelargonio G, Russo E, et al. Usefulness of intracardiac echocardiography for the diagnosis of cardiovascular implantable electronic device-related endocarditis. J Am Coll Cardiol. 2013;61(13): $1398-1405$.

30. Cheung G, Vejlstrup N, Ihlemann N, et al. Infective endocarditis following percutaneous pulmonary valve replacement: diagnostic challenges and application of intra-cardiac echocardiography. Int J Cardiol. 2013;169(6):425-429.

31. Poommipanit P, Tobis J. Intracardiac echocardiography (ICE)-guided biopsy of a right atrial mass. J Invasive Cardiol. 2011;23(5): E99-E101.

32. Kamiya K, Sakakibara M, Yamada S, et al. Diffuse large B cell lymphoma diagnosed by intracardiac echocardiography-guided cardiac tumor biopsy. Intern Med. 2012;51(9):1043-1047.

33. Kuppahally SS, Litwin SE, Michaels AD. Endomyocardial biopsy of right atrial angiosarcoma guided by intracardiac echocardiography. Cardiol Res Pract. 2010;68:1726. 
Medical Devices: Evidence and Research

Dovepress

\section{Publish your work in this journal}

Medical Devices: Evidence and Research is an international, peerreviewed, open access journal that focuses on the evidence, technology, research, and expert opinion supporting the use and application of medical devices in the diagnosis, treatment and management of clinical conditions and physiological processes. The identification of novel devices and optimal use of existing devices which will lead to improved clinical outcomes and more effective patient management and safety is a key feature. The manuscript management system is completely online and includes a quick and fair peer-review system. Visit http://www. dovepress.com/testimonials.php to read real quotes from authors.

Submit your manuscript here: http://www.dovepress.com/medical-devices-evidence-and-research-journal 